\title{
Erythropoietin with Retrobulbar Administration Protects Retinal Ganglion Cells from Acute Elevated Intraocular Pressure in Rats
}

\author{
Yi-sheng Zhong, Xiao-hong Liu, Yu Cheng, and Ying-jun Min
}

\begin{abstract}
Objective: The aim of this study was to clarify whether erythropoietin (EPO) with a retrobulbar administration could protect retinal ganglion cells (RGCs) from acute elevated intraocular pressure (IOP).

Methods: The anterior chamber of the right eye was cannulated, and the IOP was raised to $70 \mathrm{~mm} \mathrm{Hg}$ for a duration of up to $60 \mathrm{~min}$. One thousand (1000) units of recombinant erythropoietin (rhEPO) or vehicle solution was administered a retrobulbar injection immediately after the onset of the acute elevated IOP. After 1 week, RGCs were labeled with a commercially available retrograde tracer applied to the superior colliculi. Densities of surviving RGCs were estimated by counting retrograde-tracer-labeled cells in whole-mounted retinas. The ultrastructural changes of RGCs were observed by transmission electron microscope. Immunocytochemistry was used to detect EPO and erythropoietin receptor (EPOR) expression in the RGCs layer.

Results: The acute elevated IOP could result in the loss of RGCs. The number of surviving RGCs per square millimeter in the eyes of the acute elevated IOP + rhEPO retrobulbar injection group was significantly higher than that in the eyes of the acute elevated IOP and acute elevated IOP + vehicle solution retrobulbar injection groups $(P<0.05)$. The number of the organelles in the RGCs plasm decreased, but some intact mitochondrian still existed in the RGCs plasm in the eyes of the acute elevated IOP + rhEPO retrobulbar injection group. The densities of EPO and EPOR expression of the RGCs layer in the eyes of the acute elevated IOP + rhEPO retrobulbar injection group were significantly higher than that in the eyes of the acute elevated IOP and acute elevated IOP + vehicle solution retrobulbar injection groups $(P<0.01)$.

Conclusion: EPO with a retrobulbar administration could protect RGCs from acute elevated IOP.
\end{abstract}

\section{Introduction}

E RYTHROPOIETIN (EPO) HAS BEEN VIEWED TRADITIONALLY AS A hematopoietic cytokine produced by the fetal liver and adult kidney in response to hypoxia. Results of recent studies now support a physiologic role for EPO within the central nervous system (CNS). The expression of EPO and EPO receptors (EPOR) in the CNS and the upregulation of EPO by hypoxia/ischemia in vitro and in vivo suggest that this cytokine is an important mediator of the brain's response to injury. ${ }^{1-7}$

EPO could act on the CNS as a neuroprotective factor, particularly in conditions of neural damage, such as hypoxia, ischemia, or brain hemorrhage. Marked changes in EPO and
EPOR gene expression have been reported to occur in brain tissue after ischemic injury. ${ }^{8}$ In vivo evidence for a neuroprotective function of EPO has been demonstrated in different models of hypoxic-ischemic injury ${ }^{8-11}$ and in an animal model of Parkinson's disease. ${ }^{12}$ Further, EPO has been shown to protect primary cultured neurons from N-methyldaspartate (NMDA)-receptor-mediated glutamate toxicity and against ischemia-induced neuronal death. ${ }^{2,9}$ In the retina, EPO exerts its protective effects against light-induced retinal degeneration, ${ }^{13}$ retinal ischemia, ${ }^{14}$ experimental glaucoma, and axotomy-induced degeneration of retinal ganglion cells (RGCs). ${ }^{15,16}$ The EPO-administration approach, in those studies for retinal neurons' protective effects, was the intravitreal (i.t.v.) or intraperitoneal (i.p.) injection; however,

Department of Ophthalmology, Ruijin Hospital Affiliated Shanghai Jiaotong University, Shanghai, China.

None of the authors had any proprietary interest in the development and marketing of any products mentioned in this paper.

Yi-sheng Zhong and Xiao-hong Liu contributed equally to this work.

This research was supported by the Shanghai Science and Technology Committee Project Foundation (No. 04JC14023). 
the direct delivery of EPO into the vitreous capacity is not a practical approach in most clinical contexts. Meanwhile, the i.t.v. injection could result in the risk of endophthalmitis and retinal neovascularization. ${ }^{17,18}$ Retrobulbar injection is the ordinary administration approach in the clinic, and it is safer than the i.t.v. injection. However, there are no data available concerning the retinal neurons' protective effect of EPO with the retrobulbar injection. Therefore, the present study was to clarify whether EPO with a retrobulbar administration could protect RGCs from acute elevated intraocular pressure (IOP).

\section{Methods}

\section{Rat model of acute elevated IOP}

Animals used in this study were treated in accordance with the Association for Research in Vision and Ophthalmology Statement for the Use of Animals in Ophthalmic and Vision Research. Male Sprague-Dawley rats weighing approximately $250 \mathrm{~g}$ were obtained from the Experimental Animal Center of Medical College, Shanghai Jiaotong University (Shanghai, China). The rats were fed ad libitum and maintained in temperature-controlled room with a 12-h light-dark cycle. A total of 75 rats were used for this study.

The rats were anesthetized by an i.p. injection of 3\% pentobarbital sodium $(30 \mathrm{mg} / \mathrm{kg}$ ). The anterior chamber of the right eye was cannulated with a $27-\mathrm{G}$ needle attached to an infusion line of normal saline and to a manometer. The corneal puncture site was sealed with cyanoacrylate cement. The IOP was raised to $70 \mathrm{~mm} \mathrm{Hg}$ for a duration of up to $60 \mathrm{~min}$. After 60 min of acute elevated IOP, the needle was withdrawn and the IOP normalized. Erythromycin ophthalmic ointment was applied topically to the right eye before and after the cannulation of the anterior chamber. The anterior chamber of the right eye in the sham-operated group was similarly cannulated, and the IOP was raised to $15 \mathrm{~mm} \mathrm{Hg}$ for a duration of up to $60 \mathrm{~min}$.

\section{Drug administration}

For the retrobulbar drug administration recombinant erythropoietin (rhEPO; Roche Molecular Biochemicals, Mannheim, Germany) was diluted in rhEPO vehicle solution. Then $100 \mu \mathrm{L}$ (1000 units) of rhEPO or vehicle solution was administered by retrobulbar injection immediately after the onset of the acute elevated IOP. According to the drug administration, the animals were divided into five groups: the control group (untouched control), the sham-operated group, the acute elevated IOP group, the acute elevated IOP + rhEPO retrobulbar injection group, and the acute elevated IOP + vehicle solution retrobulbar injection group.

\section{Retrograde labeling of RGCs}

Six (6) rats of each group were randomly chosen for the retrograde labeling of RGCs. Three (3) days before sacrifice, the animals were anesthetized by an i.p. injection of $3 \%$ pentobarbital sodium $(30 \mathrm{mg} / \mathrm{kg})$. A bur hole was drilled into the pericranium overlying the superior colliculi $(0.7 \mathrm{~mm}$ lateral to the sagittal suture and $3 \mathrm{~mm}$ posterior to the bregma). For the retrograde labeling of RGCs, a Hamilton syringe (Hamilton Medical Systems, Inc., Reno, NV) was inserted 2.0 $\mathrm{mm}$ beneath the brain surface, and $0.7 \mu \mathrm{L}$ of Fluoro-Gold ${ }^{\mathrm{TM}}$ (Fluorochrome, Denver, CO) was injected stereotactically into both superior colliculi (infusion rate, $0.7 \mu \mathrm{L} / \mathrm{min}$ ). After infusion, the injection needle remained inside the brain for $2 \mathrm{~min}$ to prevent Fluoro-Gold diffusion along the needle track. The syringe was then withdrawn, and the wounds were carefully closed with a suture.

One (1) week after the onset of the acute elevated IOP, the animals were sacrificed by an overdose of 3\% pentobarbital sodium and whole, flat-mounted retinas were assayed for RGCs density. Six (6) eyes of each group were randomly chosen for flat mounts. The rats' eyes were enucleated and fixed in $4 \%$ paraformaldehyde for $1 \mathrm{~h}$. Eyes were bisected at the equator, the lens was removed, and the posterior segments were prepared for flat mounts. Retinas were dissected from the underlying sclera, flattened by six radial cuts, and mounted vitreal-side-up on gelatin-coated slides. Labeled RGCs were counted by using fluorescence microscopy. Noting the retinal topography, six fields in the regional areas, approximately $4.0 \mathrm{~mm}$ from the optic disc, were counted.

\section{Ultrastructural analysis of RGCs}

Three (3) rats of each group were randomly chosen for the ultrastructural analysis. The rats' eyes were enucleated and bisected at the equator, the lens was removed, and the posterior segments were prepared for the ultrastructural analysis. After $6 \mathrm{~h}$ of fixation in $2.5 \%$ glutaraldehyde, the retinas were rinsed with phosphate-buffered saline (PBS) and postfixed in 1\% $\mathrm{OsO}_{4}$ for $2 \mathrm{~h}$ at room temperature. The retinas were dehydrated in graded alcohols to $100 \%$ ethanol, then washed with $100 \%$ propylene oxide, and embedded in Spurr resin (Electron Microscopy Sciences, Fort Washington, PA). Thin sections were cut, collected on 200 mesh grids, and stained with uranyl acetate and lead citrate. The sections were examined and photographed in a Hitachi H-500 transmission electron microscope (Hitachi Ltd., Tokyo, Japan). RGCs were assessed by the same investigator in a masked fashion.

\section{Immunohistochemistry}

Six (6) rats of each group were randomly chosen for EPO and EPOR immunohistochemical staining. One (1) week after the onset of the acute elevated IOP, the animals were sacrificed by overdose of $3 \%$ pentobarbital sodium, and the eyes were quickly enucleated and chilled in iced PBS. The eyes were dissected and immediately fixed as eye cups without cornea and lens for $48 \mathrm{~h}$ in the mixed fixation solution (100$\mathrm{mL}$ fixation solution contained $40 \%$ formaldehyde $10 \mathrm{~mL}$, acetic acid $5 \mathrm{~mL}, 95 \%$ alcohol $50 \mathrm{~mL}, 25 \%$ glutaraldehyde 5 $\mathrm{mL}$, and double-distilled $\mathrm{H}_{2} \mathrm{O} 30 \mathrm{~mL}$ ) at $4^{\circ} \mathrm{C}$. All eyes were processed by paraffin embedding, and longitudinal sections $(4 \mu \mathrm{m})$ were cut through the globe along the anterior-posterior axis. Retinal sections were deparaffinized, rehydrated, and rinsed in PBS solution for $5 \mathrm{~min}$. The sections were put into the antigen activity recovery solution (citric acid/citrate sodium solution, $\mathrm{pH}$ 6.0) and heated with high fire in a microwave oven for $3 \mathrm{~min}$, then cooled at room temperature. The sections were treated with 3\% hydrogen peroxide for 10 min to block endogenous peroxidase activity. After the sections were washed with PBS solution containing $0.1 \%$ bovine serum albumin (BSA), they were incubated with inactivated normal goat serum for $30 \mathrm{~min}$ at room temperature to block background staining. The sections were then incubated with rabbit-anti-rat polyclonal antibodies against EPO (1:50; Santa 
Cruz Biotechnology, Santa Cruz, CA; sc-7956) and EPOR (1:100; Santa Cruz Biotechnology; sc-5624) overnight at $4^{\circ} \mathrm{C}$, respectively. After the sections were washed, they were incubated with the biotinylated secondary antibodies (1:400; Santa Cruz Biotechnology) for $1 \mathrm{~h}$ at room temperature and then with avidin-biotin complex (ABC solution; Santa Cruz Biotechnology) for $1 \mathrm{~h}$ at room temperature. After several washes, color was developed by incubation with 3,3-diaminobenzidine tetrahydrochloride (DAB; Sigma-Aldrich, St. Louis, MO), used as a cosubstrate for $5 \mathrm{~min}$. Sections were counterstained with hematoxylin and mounted. Absence of primary antibody and incubation with preimmune serum were used as one negative control for each antibody. Slides were examined with a microscope (Nikon, Tokyo, Japan), and images were recorded by photography. Visual assessment and densitometric measurement, using an image plus 4 program (Axioskop 2 plus; Zeiss Inc., Göttingen, Germany) determined the staining density of the RGCs layer. For each sample, three different fields, approximately $4.0 \mathrm{~mm}$ from the optic disc, were chosen to detect the staining density at a final magnification of $400 \times$.

\section{Statistical analysis}

Data are expressed as mean \pm standard deviation, unless otherwise stated. Statistical analyses were performed by using the SPSS for Windows version 10.0 software package (SPSS, Inc., Chicago, IL). To compare data among three or more groups, one-way analysis of variance (ANOVA), followed by either least significant difference test (homogeneity of variance) or the Games-Howell test (nonhomogeneity of variance), was used. A value of $P<0.05$ was considered to be statistically significant.

\section{Results}

\section{Effect of EPO on RGCs survival}

In retinas, the only cells that appeared to be labeled with Fluoro-Gold were RGCs. These were identified by the typical punctuate fluorescence present in the somata (Fig. 1). All quadrants of all retinas were successfully labeled.

Figure 2 showed the densities of surviving RGCs in the eyes of each group. The number of surviving RGCs per square millimeter was $2442 \pm 88(n=6)$ in the eyes of the control group and $2427 \pm 103(n=6)$ in the eyes of the shamoperated group (Fig. 2; $P>0.05$ ). The acute elevated IOP could result in the loss of RGCs. The numbers of surviving RGCs per square millimeter in the eyes of the acute elevated
IOP group $(1733 \pm 62 ; n=6)$, the acute elevated IOP + rhEPO retrobulbar injection group $(2142 \pm 98 ; n=6)$, and the acute elevated IOP + vehicle solution retrobulbar injection group (1724 $\pm 102 ; \mathrm{n}=6)$, were significantly lower than that in the eyes of the control and sham-operated groups $(F=45.48, P<0.05)$. However, the number of surviving RGCs per square millimeter in the eyes of the acute elevated $\mathrm{IOP}+$ rhEPO retrobulbar injection group was significantly higher than that in the eyes of the acute elevated IOP and acute elevated IOP + vehicle solution retrobulbar injection groups (Fig. 2; $P<0.05$ ).

\section{Effect of rhEPO on RGCs ultrastructure}

Transmission electron microscopy (TEM) revealed the abundant organelles in the RGCs plasm in the eyes of the control (Fig. 3A) and sham-operated groups. The number of the organelles in the RGCs plasm appeared to decrease, and some vacuolated mitochondrian existed in RGCs splasm in the eyes of the acute elevated IOP (Fig. 3B) and acute elevated IOP + vehicle solution retrobulbar injection groups. The number of the organelles in the RGCs plasm also decreased, but some intact mitochondrian still existed in the RGCs plasm in the eyes of the acute elevated IOP + rhEPO retrobulbar injection group (Fig. 3C).

\section{EPO/EPOR expression in RGC layer}

A moderate EPO and EPOR immunoreactivity staining was observed in the RGCs layer in the eyes of the control (Fig. 4A and 4D) and sham-operated groups. The densities of EPO and EPOR expression in the RGCs layer increased in the eyes of the acute elevated IOP group (Fig. $4 \mathrm{~B}$ and $4 \mathrm{E}$ ), the acute elevated IOP + rhEPO retrobulbar injection group (Fig. 4C and 4F), and the acute elevated IOP + vehicle solution retrobulbar injection group (Table 1). The densities of EPO and EPOR expression of the RGCs layer in the eyes of the acute elevated IOP + rhEPO retrobulbar injection group were significantly higher than that in the eyes of the acute elevated IOP and acute elevated IOP + vehicle solution retrobulbar injection groups (Table 1 ).

\section{Discussion}

To gain a deeper understanding of the nature of EPO-dependent neuroprotection, we studied whether rhEPO with a retrobulbar administration could protect RGCs from suffering from acute elevated IOP. Our results suggested that the densities of surviving RGCs in the eyes of the acute elevated
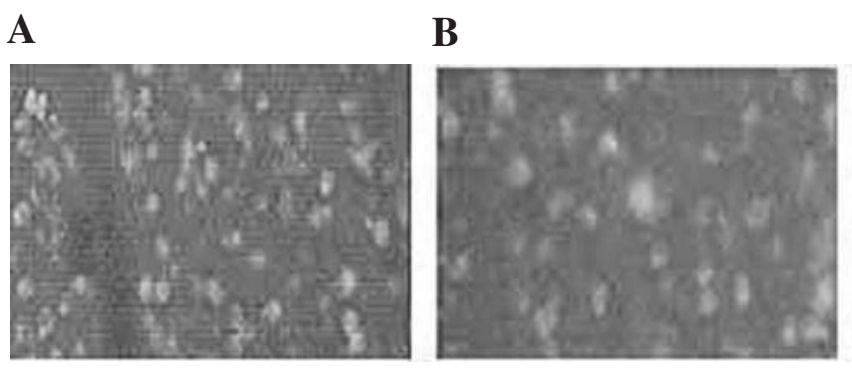

C

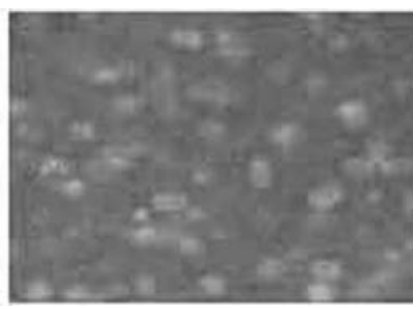

FIG. 1. Retrogradedly-labeled retinas with normal intraocular pressure (IOP) and acute elevated IOP, with and without treatment with a recombinant erythropoietin (rhEPO) retrobulbar administration. (A) Control. (B) Vehicle-treated. (C) rhEPO-treated. 


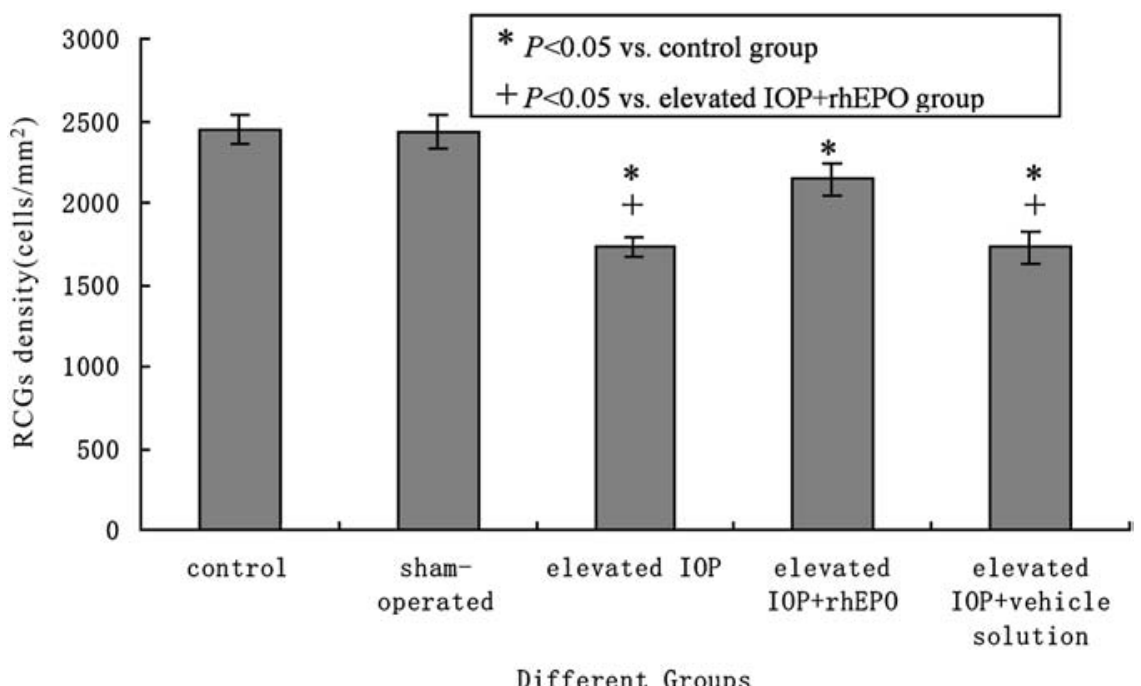

FIG. 2. The densities of surviving retinal ganglion cells (RGCs) in the eyes of each group. RGCs were counted in the retina, approximately $4.0 \mathrm{~mm}$ from the optic disc. The densities of surviving RGCs in the eyes of the acute elevated intraocular pressure (IOP) group, the acute elevated IOP + recombinant erythropoietin (rhEPO) retrobulbar injection and acute elevated IOP + vehicle solution retrobulbar injection groups were significantly lower than that in the eyes of the control and sham-operated groups $(F=45.48, P<0.05)$. The densities of surviving RGCs in the eyes of the acute elevated IOP + rhEPO retrobulbar injection group was significantly higher than that in the eyes of the acute elevated IOP and acute elevated IOP + vehicle solution retrobulbar injection groups $(P<0.05)$.

$\mathrm{IOP}+$ rhEPO retrobulbar injection group was lower than that in the eyes of the control group, but it was significantly higher than that in the eyes of the acute elevated IOP and acute elevated IOP + vehicle solution retrobulbar injection groups, and the ultrastructure of RGCs improved. The results indicated that the acute elevated IOP could result in the loss of RGCs, and rhEPO with a retrobulbar administration was able to protect RGCs.

Multiple mechanisms may explain how EPO protects neurons from acute injury. The systemic administration of EPO significantly reduces TUNEL-positive cells in the ischemic penumbra after focal cerebral ischemia, suggesting an antiapoptotic effect. ${ }^{7}$ Similarly, ischemic retinas that had received EPO exhibited a marked decrease in TUNEL labeling. ${ }^{14}$. EPO may inhibit neuronal apoptosis by upregulating Bcl-xL and Bcl-2. ${ }^{19} \mathrm{EPO}$ also has been shown to inhibit the apoptosis of cultured cortical neurons deprived of growth factors or exposed to kainic acid by activating mitogen-activated protein kinase and phosphatidylinositol 3-kinase/AKt pathways. ${ }^{7}$ These signaling mechanisms participate in the antiapoptotic effect of brain-derived neurotrophic factor, which protects retinal neurons from a variety of different injuries. ${ }^{20} \mathrm{EPO}$, therefore, may be a particularly effective agent for retinal disorders. EPO also may exert an antiapoptotic effect by recruiting nuclear factor kappa-beta (NF-kB), ${ }^{21}$ which translocates to the nucleus and can activate neuroprotective genes, such as superoxide dismutase or inhibitors of apoptosis proteins. Cytoprotection by EPO was also closely related to the maintenance of the mitochondrial membrane potential. The loss of membrane potential through the opening of the mitochondrial permeability transition pore represents a significant determinant for cell injury and the subsequent induction of apoptosis. ${ }^{22,23}$ Studies that involve free-radical injury, anoxia, or oxygen-glucose deprivation demonstrate
A

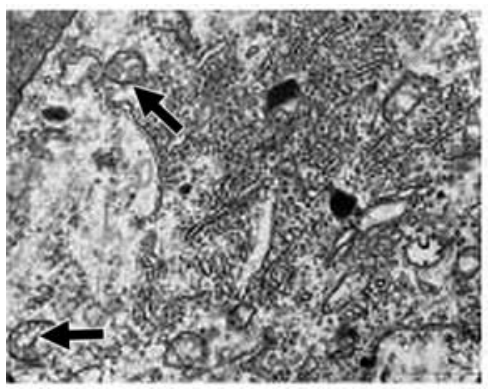

B

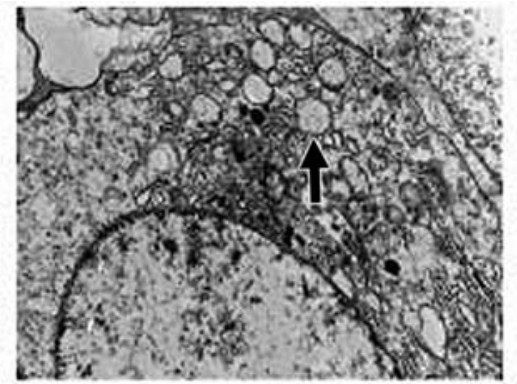

C

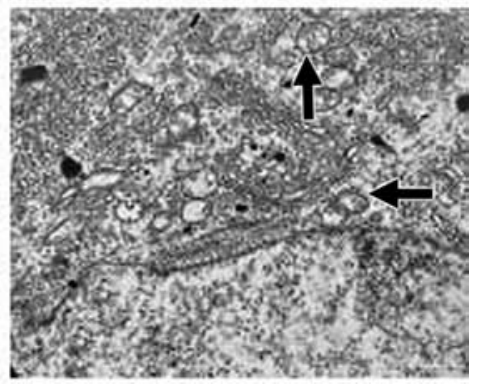

FIG. 3. The ultrastructural images of retinal ganglion cells (RGCs). (A) The abundant organelles appeared in the RGC plasm, and the intact mitochondrion (black arrow) existed in RGCs plasm in the eyes of the control group. (B) The number of the organelles in the RGCs plasm appeared to decrease, and the mitochondrial crista disappeared, and some vacuolated mitochondrian (black arrow) existed in RGCs plasm in the eyes of the acute elevated IOP group. (C) The number of the organelles in the RGCs plasm decreased, but some intact mitochondrian (black arrow) still existed in the RGCs plasm in the eyes of the acute elevated IOP + rhEPO retrobulbar injection group (TEM; 17,500×). 
A
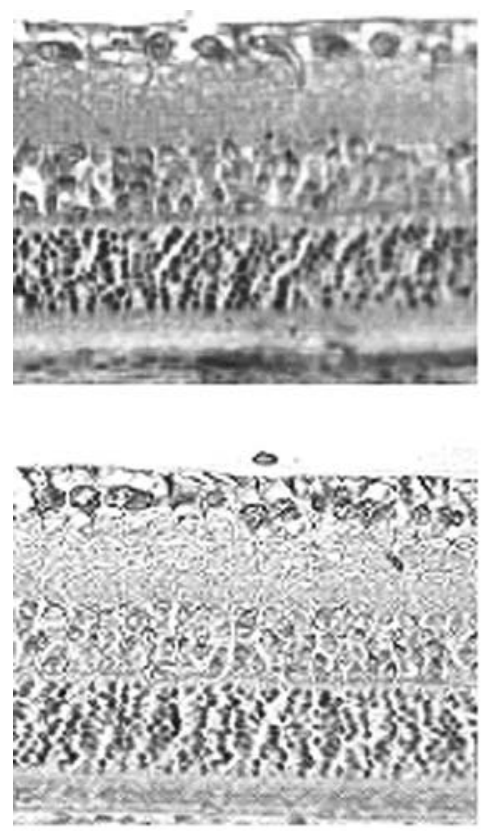

D
B
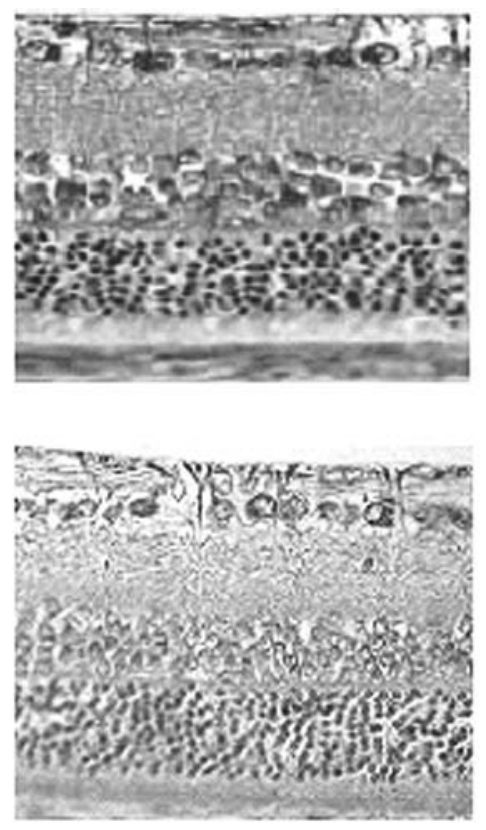

$\mathbf{E}$
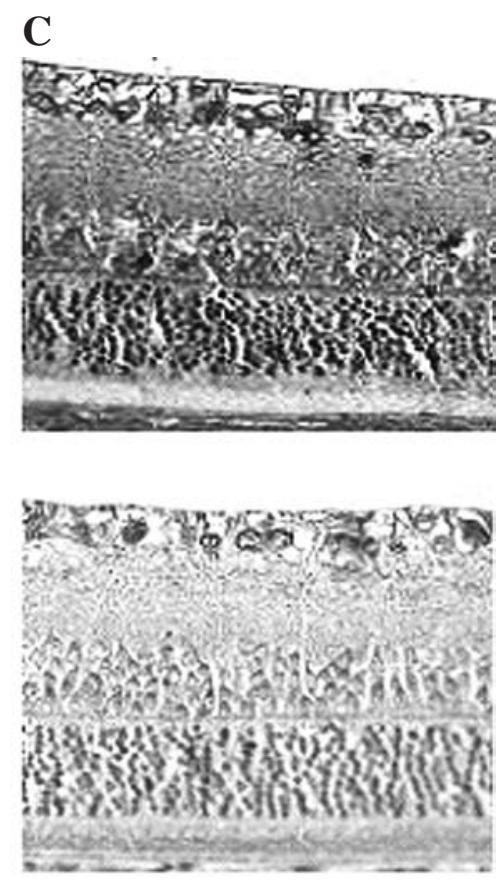

$\mathbf{F}$

FIG. 4. The immunohistochemical staining images of erythropoietin (EPO) and erythropoietin receptor (EPOR). A moderate EPO (A) and EPOR (D) immunoreactivity staining was observed in the RGC layer in the eyes of the control group; a moderate-to-strong EPO (B) and EPOR (E) immunoreactivity staining was observed in the RGCs layer in the eyes of the acute elevated IOP group; a moderate-to-strong EPO (C) and EPOR (F) immunoreactivity staining was observed in the RGC layer in the eyes of the acute elevated IOP + rhEPO retrobulbar injection group (immunohistochemistry staining, $400 \times$ ).

that EPO prevents the depolarization of the mitochondrial membrane not only by maintaining membrane potential and preventing the release of cytochrome c, but also by using Akt and regulating caspase-mediated pathways. ${ }^{24,25}$

A full understanding of how EPO mediates its effects across the blood-brain barrier (or blood-retina barrier) has not yet been elucidated. Because cerebral injury can impair the integrity of the blood-brain barrier, it is hypothesized that EPO acquires access to cerebral tissue through regions of the compromised blood-brain barrier. ${ }^{10,26,27}$ Although this mechanism certainly could contribute to the site-specific delivery of EPO after an injury, it cannot explain the protective effects of 24-h pretreatment in the middle cerebral artery occlusion and kainate models, for which the serum EPO levels were low at the time of injury. ${ }^{10}$ Further, previous studies demonstrated that EPO prevents blood-brain barrier permeability during injury and maintains the establishment of cell-to-cell junctions, and can ulti- mately assist with the repair of the blood-brain barrier. ${ }^{27}$ More importantly, EPO provides direct cerebral endothelial cells' protection and protects against nuclear degeneration during oxidative stress. ${ }^{28-30}$ Brines et al. ${ }^{10}$ found that EPOR was presented on the capillaries of the brain, including the endothelial cells, but excluded from larger vessels, and the biotin-labeled EPO only surrounded the blood-brain barrier capillaries and not around larger vessels, as would be expected for a specific receptor-mediated translocation of EPO into the brain.

The expression of both EPO and EPOR is greatly enhanced by hypoxia in neurons and astroglial cells. ${ }^{8}$ In most tissues, including the brain, the hypoxia-dependent expression of EPO and EPOR is regulated primarily by hypoxia-inducible factor 1 (HIF-1), an $\alpha, \beta$-heterodimeric protein that is activated by a variety of stressors, such as hypoxia. HIF-1 appears to have an important role in the regulation of the expression of EPO and EPOR to foster protection against hypoxic cell injury. ${ }^{31}$ The

Table 1. The Expression Gray Values of EPO and EPOR ImmunOREactivity in the Retinal Ganglion Cell Layer $(\chi \pm \mathrm{SD}, N=6)$

\begin{tabular}{lcc}
\hline Groups & EPO expression & EPOR expression \\
\hline Control & $145.88 \pm 7.21$ & $138.41 \pm 9.28$ \\
Sham-operated & $145.06 \pm 9.90$ & $137.30 \pm 11.21$ \\
Acute elevated IOP & $164.22 \pm 5.13^{\mathrm{a}}$ & $156.97 \pm 13.69^{\mathrm{a}}$ \\
Acute elevated IOP + rhEPO & $180.83 \pm 8.71^{\mathrm{a}, \mathrm{b}}$ & $174.63 \pm 9.56^{\mathrm{a}, \mathrm{b}}$ \\
Acute elevated IOP + vehicle solution & $163.91 \pm 5.74^{\mathrm{a}}$ & $164.24 \pm 9.86^{\mathrm{a}}$ \\
$F$ & 23.40 & 13.53 \\
$P$ & $<0.01$ & $<0.01$ \\
\hline
\end{tabular}

aRepresents $P<0.05$ versus control group.

${ }^{b}$ Represents $P<0.05$ versus the acute elevated IOP and acute elevated IOP + vehicle solution groups. 
present study showed that the densities of EPO and EPOR expression in the RGCs layer increased in the eyes of the acute elevated IOP group, the acute elevated IOP + rhEPO retrobulbar injection group, and the acute elevated IOP + vehicle solution retrobulbar injection group, which was consistent with the upregulation of EPO and EPOR expression by hypoxia/ischemia in neurons. ${ }^{8}$ Our results also showed that the densities of EPO and EPOR expression of the RGCs layer in the eyes of the acute elevated IOP + rhEPO retrobulbar injection group were significantly higher than that in the eyes of the acute elevated IOP and acute elevated IOP + vehicle solution retrobulbar injection groups, which may be attributed to the endogenous EPO and EPOR expression upregulation by exogenous EPO or a specific receptor-mediated translocation of EPO into the retina. Further work is obviously needed to evaluate these complex interactions in vivo.

The dosage of rhEPO we administered in this study was chosen according to a previous study showing that a $1000-5000-\mathrm{U} / \mathrm{kg}$ rhEPO i.p. injection in the light-induced retinal degeneration model produced a protection effect of the photoreceptor. ${ }^{13}$ The quantity of rhEPO in the present study was higher than that needed for erythropoiesis and substantially higher than most conventional clinical dosages. Nonetheless, large doses of rhEPO have been tested in preclinical and phase I clinical trials without adverse effects, 32,33 and many cancer patients now receive rhEPO as a weekly injection of 40,000-60,000 units.

\section{Conclusion}

In conclusion, the results of the present study constitute a basis for examining the effectiveness of rhEPO with the retrobulbar administration for the treatment of retinal neuropathy that is currently therapeutically underserved. However, further investigations are need to demonstrate the protection mechanisms and the side effects of retrobulbar administration of rhEPO.

\section{References}

1. Genc, S., Koroglu, T.F., and Genc, K. Erythropoietin and the nervous system. Brain Res. 1000:19-31, 2004.

2. Morishita, E., Masuda, S., Nagao, M., et al. Erythropoietin receptor is expressed in rat hippocampal and cerebral cortical neurons, and erythropoietin prevents in vitro glutamateinduced neuronal death. Neuroscience 6:105-116, 1997.

3. Dame, C., Bartmann, P., Wolber, E.M., et al. Erythropoietin gene expression in different areas of the developing human central nervous system. Dev. Brain Res. 125:69-74, 2000.

4. Digicaylioglu, M., Bichet, S., Marti, H.H., et al. Localization of specific erythropoietin binding sites in defined areas of the mouse brain. Proc. Natl. Acad. Sci. USA. 92:3717-3720, 1995.

5. Bernaudin, M., Bellail, A., Marti, H.H., et al. Neurons and astrocytes express Epo mRNA: Oxygen-sensing mechanisms that involve the redox state of the brain. Glia 30:271-278, 2000.

6. Liu R, Suzuki A, Guo Z, Mizuno Y, Urabe T. Intrinsic and extrinsic erythropoietin enhances neuroprotection against ischemia and reperfusion injury in vitro. J. Neurochem. 96: 1101-1110, 2006.

7. Siren, A.L., Fratelli, M., Brines, M., et al. Erythropoietin prevents neuronal apoptosis after cerebral ischemia and metabolic stress. Proc. Natl. Acad. Sci. U.S.A. 98:4044-4049, 2001.

8. Bernaudin, M., Marti, H.H., Roussel, S., et al. A potential role for erythropoietin in focal permanent cerebral ischemia in mice. J. Cereb. Blood Flow Metab. 19:643-651, 1999.
9. Sakanaka, M., Wen, T.C., Matsuda, S., et al. In vivo evidence that erythropoietin protects neurons from ischemic damage. Proc. Natl. Acad. Sci. U.S.A. 95:4635-4640, 1998.

10. Brines, M.L., Ghezzi, P., Keenan, S., et al. Erythropoietin crosses the blood-brain barrier to protect against experimental brain injury. Proc. Natl. Acad. Sci. U.S.A.. 97:1052610531, 2000.

11. Calapai, G., Marciano, M.C., Corica, F., et al. Erythropoietin protects against brain ischemic injury by inhibition of nitric oxide formation. Eur. J. Pharmacol. 401:349-356, 2000.

12. Masuda, S., Nagao, M., and Sasaki, R. Erythropoietic, neurotrophic, and angiogenic functions of erythropoietin and regulation of erythropoietin production. [review]. Int. J. Hematol. 70:1-6, 1999.

13. Grimm, C., Wenzel, A., Groszer, M., et al. HIF-1-induced erythropoietin in the hypoxic retina protects against lightinduced retinal degeneration. Nat. Med. 8:718-724, 2002.

14. Junk, A.K., Mammis, A., Savitz, S.I., et al. Erythropoietin administration protects retinal neurons from acute ischemiareperfusion injury. Proc. Natl. Acad. Sci. U.S.A.. 99:1065910664, 2002.

15. Tsai, J.C., Wu, L., Worgul, B., et al. Intravitreal administration of erythropoietin and preservation of retinal ganglion cells in an experimental rat model of glaucoma. Curr. Eye Res. 30:1025-1031, 2005.

16. Kilic, Ü., Kilic, E., Soliz, J., et al. Erythropoietin protects from axotomy-induced degeneration of retinal ganglion cells by activating ERK-1/-2. FASEB J. 19:249-251, 2005.

17. Heeschen, C., Aicher, A., Lehmann, R., et al. Erythropoietin is a potent physiologic stimulus for endothelial progenitor cell mobilization. Blood 102:1340-1346, 2003.

18. Jaquet, K., Krause, K., Tawakol-Khodai, M., et al. Erythropoietin and VEGF exhibit equal angiogenic potential. Microvasc Res. 64:326-333, 2002.

19. Wen, T.C., Sadamoto, Y., Tanaka, J., et al. Erythropoietin protects neurons against chemical hypoxia and cerebral ischemic injury by upregulating Bcl-xL expression. J. Neurosci. Res. 67:795-803, 2002.

20. Klocker, N., Kermer, P., Weishaupt, J.H., et al. Brain-derived neurotrophic factor-mediated neuroprotection of adult rat retinal ganglion cells in vivo does not exclusively depend on phosphatidyl-inositol-3'-kinase/protein kinase B signaling. J. Neurosci. 20:6962-6967, 2000.

21. Digicaylioglu, M., and Lipton, S.A. Erythropoietin-mediated neuroprotection involves cross-talk between Jak2 and NF-kappaB signaling cascades. Nature 412:641-647, 2001.

22. Ekshyyan, O., and Aw, T.Y. Apoptosis in acute and chronic neurological disorders. Front Biosci. 9:1567-1576, 2004.

23. Maiese, K., and Chong, Z.Z. Insights into oxidative stress and potential novel therapeutic targets for Alzheimer's disease. Restor. Neurol. Neurosci. 22:87-104, 2004.

24. Kang, J.Q., Chong, Z.Z., and Maiese, K. Akt1 protects against inflammatory microglial activation through maintenance of membrane asymmetry and modulation of cysteine protease activity. J. Neurosci. Res. 74:37-51, 2003.

25. Kang, J.Q., Chong, Z.Z., and Maiese, K. Critical role for Akt1 in the modulation of apoptotic phosphatidylserine exposure and microglial activation. Mol. Pharmacol. 64: 557-569, 2003.

26. Grasso, G., Buemi, M., Alafaci, C., et al. Beneficial effects of systemic administration of recombinant human erythropoietin in rabbits subjected to subarachnoid hemorrhage. Proc. Natl. Acad. Sci. U.S.A. 99:5627-5631, 2002.

27. Martínez-Estrada, O.M., Rodríguez-Millán, E., González-De Vicente, E., et al. Erythropoietin protects the in vitro blood- 
brain barrier against VEGF-induced permeability. Eur. J. Neurosci. 18:2538-2544, 2003.

28. Chong, Z.Z., Kang, J.Q., and Maiese, K. Erythropoietin is a novel vascular protectant through activation of Akt1 and mitochondrial modulation of cysteine proteases. Circulation 106:2973-2979, 2002

29. Chong, Z.Z., Kang, J.Q., and Maiese, K. Angiogenesis and plasticity: Role of erythropoietin in vascular systems. J. Hematother. Stem Cell Res. 11:863-871, 2002.

30. Chong, Z.Z., Kang, J.Q., and Maiese, K. Apaf-1, Bcl-xL, cytochrome $c$, and caspase- 9 form the critical elements for cerebral vascular protection by erythropoietin. J. Cereb. Blood Flow Metab. 23:320-330, 2003.

31. Heidbreder, M., Fröhlich, F., Jöhren, O., et al. Hypoxia rapidly activates HIF-3alpha mRNA expression. FASEB J. 17:1541-1543, 2003.

32. Cheung, W.K., Goon, B.L., Guilfoyle, M.C., et al. Pharmacokinetics and pharmacodynamics of recombinant human erythropoietin after single and multiple subcutaneous doses to healthy subjects. Clin. Pharmacol. Ther. 64:412-423, 1998.

33. Ehrenreich, H., Hasselblatt, M., Dembowski, C., et al. Erythropoietin therapy for acute stroke is both safe and beneficial. Mol. Med. 8:495-505, 2002.

Received: February 17, 2008

Accepted: May 21, 2008

Reprint Requests: Yi-sheng Zhong Department of Ophthalmology Ruijin Hospital Affiliated Shanghai Jiaotong University 197 Ruijin No. 2 Road Shanghai 200025

China

E-mail: yszhong68@yahoo.com.cn 
